\title{
Mobbing: From a Social Phenomenon to Psychopathology: Preliminary Data
}

\author{
Diana Galletta $^{1^{*}}$, Gianpiero Sica ${ }^{2}$, Annamaria Califano $^{3}$, Cecilia Aurino ${ }^{3}$, Pierpaolo Di Lorenzo ${ }^{4}$ and Claudio Buccelli ${ }^{4}$ \\ ${ }^{1}$ Division of Clinical Psycho diagnostics and Neuropsychology "Federico II" University of Naples, Italy \\ ${ }^{2}$ Department of Neurosciences, "Federico II" University of Naples, Italy \\ ${ }^{3}$ Psychologist Department of Neurosciences, "Federico II" University of Naples, Italy \\ ${ }^{4}$ Department of Public Medicine and Social Security, "Federico II" University of Naples, Italy \\ "Corresponding author: Diana Galletta, Department of Neurosciences, "Federico II" University of Naples, School of Medicine, via Pansini n. 5, CAP 80131, Naples, \\ Italy, Tel: 393396515093; E-mail: gallettad@email.it
}

Received date: 10 February, 2014, Accepted date: 28 July, 2014, Published date: 7 August, 2014

Copyright: (c) 2014 Diana Galletta, et al. This is an open-access article distributed under the terms of the Creative Commons Attribution License, which permits unrestricted use, distribution, and reproduction in any medium, provided the original author and source are credited.

\begin{abstract}
Mobbing is an increasingly frequent phenomenon that characterizes the dynamics of social and working fields. It is generally known as a hostile and vexatious behaviour perpetrated over a long period of time with the intent to isolate and oust. The aim of this study is to investigate the personality characteristics and psychopathological implications of a sample of individuals who are victims of mobbing behaviours at work. 24 adult subjects were assessed using the Minnesota Personality Inventory Second Edition (MMPI-2) in order to correlate the personality characteristics with psychopathological aspects of their own experiences. The evaluation process took place at the Psycho diagnostics and Neuroscience Unit of the "Federico II" University of Naples, between 2009 and 2011. The highest mean scores were observed at the Hypochondria $(H s=69.58)$, Hysteria $(H y=68)$ and Depression $(D=$ 69.91) scales. The Content Scales values, affecting the field of somatization, show high scores at the Anxiety scale $(\mathrm{ANX}=65.12)$ and the Health Concerns scale (HEA $=65.25)$. The results of our preliminary study suggest a close correlation between work, environmental conditioning and the onset of psychopathological disorders of the neurotic area. In addition, our sample was further tested in order to find out possible simulations or exaggerations in the final results.
\end{abstract}

Keywords: Mobbing; Personality; Simulation; Assessment; MMPI-2

\section{Introduction}

The English word "mobbing" originates from the verb "to mob" which literally means "to crowd round (someone) or into (a place) in an unruly way". Another definition of the term can be "to attack in large numbers; overwhelm". This term is also used in the ethological studies to describe the behavior of some animal species, some kind of birds in particular, when they feel the presence of a predator or a danger: in these conditions the animals tend to group together in order to surround and attack a predator (or other source of threat) to definitively drive it off.

This term was then used, at the beginning of the 70 's, by the Swedish Heinemann (1972) in order to describe different kind of negative interactions among children, in the specific case of a group that uses threat or intimidation against an individual.

Mobbing, using the definition by Leymann and Gustafsson in 1984, is "A kind of psychological terrorism that involves a hostile and nonethic attitude applied systematically - not occasionally - by a single person or more against an individual that is, from that moment on, the object of a series of vexatious and persecutory actions." These actions must recur frequently (at least once a week according to statistics) and must last for a long period of time (at least six months) [1].
As these hostile behaviours are shown fairly regularly, this kind of abuse causes significant mental illnesses, psychosomatic and social disorders.

In Italy, in 2002, the phenomenon of moral or psychic violence on the workplace is described by the Commissione Presidenza del Consigliodei Ministri (Dipartiment odella Funzione Pubblica) as follows: "Actions, behaviours or attitudes of moral or psychic violence on the workplace, repeated in time systematically or regularly, that lead to a deterioration of the working conditions and that prejudice workers' health, professional competences or dignity."

Some researches tend to identify other possible factors that help the onset of such phenomenon besides the workplace quality. As an example, Zapf declares that, among mobbing victims, groups of people also exist, suffering from anxiety or depressive disorders that are preexistent conditions; these people show deficiencies in their social behaviour, especially with their colleagues and this makes them more exposed to the risk of becoming victims in their working environment [2].

Such characteristics expose them to different problems and conflict on the workplace and could become concomitant cause of mobbing; for these reasons the personality of these workers' various aspects must be considered as an active factor that intervenes in the rising of mobbing actions ${ }^{1}$.

I. Giorgi, P. Argentero, W. Zanaletti, S.M. Candura, Un modello di valutazionepsicologica del mobbing, G. Ital. Med. Lav. Erg. 2004; $26: 2,127-132$ 
According to these theories, in the scientific community it is now widespread the awareness that, analyzing this disorder, the personality of the subject cannot be ignored.

According to Matthiesen SB and Einarsen's study (2001) the elevated 3-2-1 personality profile was most typical. Surprisingly, the victims of the common cluster reported the highest level of exposure to bullying, suggesting a vulnerability factor among the other victims.

Gandolfo R. reported a high level on scale 6 of the MMPI-2. Therefore, he stated that the group of patients who was victim of harassment behaviours showed oversensitive, suspicious and angry traits of personality when compared to the control group [3].

A sample of 146 individuals exposed to mobbing was assisted in order to analyze their personality and psychopathological profiles. The results showed: depressed mood, difficulty in making decisions, changed-related anguish and passive-aggressive traits. Moreover subjects presented somatic symptoms and need for attention and affection.

In a large sample of 733 workers who approached the Work Psychopathology Medical Centre of the Department of Mental Health of Naples [4] the most frequent psychiatric diseases related to harassment at the workplace were depression and PTSD.

One of the most comprehensive studies relating mobbing at work and psychopathological profiles was carried out by Balducci et al. [5].

In line with previous research, the results showed that the MMPI-2 mean profile was characterized by a neurotic component as evidenced by elevations of Scales 1, 2, and 3 and a paranoid component as indicated by elevation of Scale 6 .

Considering this statements, this study is based on the Minnesota Multiphasic Personality Inventory (MMPI-2) particularly useful to identify and assess the main characteristics of the examined sample. Mobbing, as a continued and persisted attack against victim's selfesteem and self-confidence, in order to cause his isolation and his exclusion from the group he works with, (Field, 1996) ${ }^{2}$ is closely related to juridical issues. From a legal point of view the worker may claim for compensation based on the quantification of the biological damage of psychic nature, when the connections between the damaging action and the actual damage really exist. Since mobbing is half-way between forensic and clinical ambits, the presence of a variety of behaviors of people who only want to satisfy personal interests cannot be ignored.

Considering this possibility, the examined sample has been tried to a close assessment in order to find out possible simulations or exaggerations in the final results.

For this reason, it has been decided to eliminate from this sample people who made highest scores, in order to make the test more homogeneous.

In that specific case, the simulation during the MMPI-2 has been inquired starting from the work of Rogers et al. (2003) making a comparison of four basic strategies: a) analysis of rare symptoms, b) analysis of symptoms' seriousness, c) analysis of evident symptoms and latent symptoms, d) analysis of symptoms' selectivity.

\footnotetext{
${ }^{2}$ Field T, Bully in sight: how to predict, resist, challenge and combat workplace bullyng, Succes Unlimited, Didcot,1996
}

These strategies make use of widely standardized validity scales supported by many studies but they also use other simulation markers that, even if they are not supported by a sufficient empirical support, they give a qualitative contribution to the whole evaluation.

\section{Materials and Methods}

The examined population in this study is made of 24 adult subjects (16 males, 8 females), all of them patients at the Psycho diagnostics and Neuroscience Unit of the "Federico II" University of Naples in Italy.

As they all declared themselves mobbing victims on their workplace they started a series of psycho diagnostic tests in order to identify some specific aspects of their personality and to analyze psychopathological aspects of their lives.

Every patient stood the Minnesota Multiphasic Personality Inventory - Second Edition (MMPI-2), one of the most popular and widely acknowledged tests to evaluate the personality characteristics in psycho diagnostics.

The Minnesota Multiphasic Personality Inventory - Second Edition (MMPI-2) is a wide-ranging test created to identify the principal structural attitudes of personality and emotional disorders.

It is a self-made personality questionnaire made of 567 items, with "True/False" answers, about different areas of the "functioning" of the person. The MMPI-2 elaboration started in the late 30's from the work of S. Hathaway and J.C. McKinley. They wanted to create a useful and intuitive test that would help psychiatrists identify psychopathological conditions. Different revisions have been made over the years making the test more topical both in a social and cultural way. The changes concern form and content of the items, but also the terminology itself has been modified in order to make it closer to the terms in use at present. In its complete form the questionnaire is correlated to: -7 Validity Scales: Cannot say scale, (L) Lie scale, (F) infrequency scale, (K) Correction scale, (Fb) Back infrequency, (Vrin) Variable Response, (Trin) Inconsistency Scale;-10 Basic clinical Scales: (Hs) Hypochondriasis, (D) Depression, (Hy) Hysteria, (Pd) Psychopathic Deviate, (Mf) Masculinity/Femininity, (Pa) Paranoia, (Pt) Psychasthenia, (Sc) Schizophrenia, (Ma) Hypomania, (Si) Social introversion.-15 Content Scales: (Anx) Anxiety, (Frs) Fears, (Obs) Obsession, (Dep) Depression, (Hea) Health worries, (Biz) Bizarre ideas, (Ang) Anger, (Cyn) Cynicism, (Asp) Antisocial behaviours, (Tpa) Type A, (Lse) Low self-esteem, (Sod) Social discomfort, (Fam) Family problems, (Wrk) Working difficulties, (Trt) Treatment resistance;-15 Supplementary Scales: (MAC-R) MacAndrew Alcoholism Scale Revised, (APS) Addictions Potential Scale, (AAS)Addictions Acknowledgement Scale (MDS)Marital Distress Scale, (O-H)Over-Controlled Hostility Scale, (Do) Dominance, (Re) Social Responsibility, (PK) Post-traumatic disorder (Keane), (A) Anxiety, (R) Repression, (Es) Ego Strength Scale , (PS) Posttraumatic disorder (Schlenger) (Mt) College Maladjustment, (Gm) Gender role male, (Gf) Gender role female.

It is well know that the Minnesota Multiphasic Personality Inventory - 2 (MMPI-2) is a self-report test. This implies that the validity of every single obtained profile depends only on the willingness of the individual to be sincere. People can have several 
reasons to lie especially when they are tested about personality and above all when the test is presented as a mean to evaluate them.

The MMPI- 2 is the most used test also in the forensic ambit and, as we said before, the examiner absolutely can't ignore the possibility that the answers of his patient could be unreliable and that he could also overstate his disorder.

In this work, a large part has been dedicated to enquire about the sample authenticity putting the MMPI-2 to 4 evaluation steps:

1) Analysis of rare symptoms using the scale F, Fb, Fp (Arbisi and Ben Porath 1995); the simulation could be probable if the final scores present these values $(\mathrm{F}>71 \mathrm{~T}, \mathrm{Fb}<90 \mathrm{~T}, \mathrm{Fp}>100 \mathrm{~T}$, Vrin $<80 \mathrm{~T}$, Trin $<$ $80 \mathrm{~T})[6,7]$.

2) Analysis of symptoms' seriousness, verified with the help of the critical items of MMPI-2 the idea is that the simulators would obtain a very high number of critical items, exceeding the expected cut off $[8,9]$.

3) Analysis of evident symptoms and latent symptoms comparing obvious items with a high value and keen items with a low value. Following this strategy the simulator would obtain high scores using the obvious items that are connected to the pathology expressed in the question and lower scores using the keen items that are not easily connectible to the construction (evident scales $=\mathrm{T}>80$ latent scales $=\mathrm{T}<50)[10]$.

4) Analysis of symptoms' selectivity using the index of simulation F-K (cut-off: 15) and the Ds scale (Greene 2000) made up to evaluate the erroneous illness stereotypes $[11,12]$.

Profiles with non-selective symptoms which are for this reason not real, score in most cases very high results in every clinical scale.

On the basis of these methods of evaluation, the examiner couldn't find any individual with exaggerated groups of symptoms and it has been possible to delineate the personality profile of all the 24 people of the sample starting from their results on the MMPI-2.

\section{Results and Conclusions}

Considering the results of the examined sample, the MMPI-2 Clinical Scales that score higher than others, are Hypochondria, Depression and Hysteria (Hs, D, Hy) (Table 1). These values constitute the so-called "neurotic triad" indicative of a person that manifest symptoms concerning the thymic axis and that are expressed by somatization processes, especially involving the body.

\begin{tabular}{|l|l|l|}
\hline Basic Scale & Mean Value & Standard Deviation \\
\hline Hs & 69.58 & 13.87 \\
\hline Hy & 68 & 12.73 \\
\hline D & 69.91 & 15.14 \\
\hline
\end{tabular}

Table 1: Basic Scales with a mean value above the cut-off.

The Hypochondria scale is composed of 32 items that are related to a broad range of physic symptomatology in addition to Depression, Hysteria and Psychasthenia scales (Pt). All of these scales principally describe: general decease, weariness, pains, breathing problems, dizziness, sensorial difficulties, difficulties of falling asleep, illness. All the subjects analyzed in the sample present: several physical symptoms, lack of attention and efficiency, worries about their health (this is evident reading the average value of the Scale of health worries HEA), pessimism and cynicism, hostility manifestations in a roundabout way. The Depression scale (D) is composed of 57 items and it helps to measure the symptomatic depression: discontent, low self-esteem, discouraged view of future. The examined subjects are often unhappy, pessimist, dysphoric, worried, self-critical, and hesitant. They often feel weak and useless. The Hysteria scale (Hy) is composed of 60 items that describe specific physical deceases or nervous excitements. Many items also imply the negation of psychological problems and lack of social anxiety. The examined subjects also tend to manifest physical symptoms as a reaction to various forms of stress (headache, asthenia, tachycardia, etc.) They also show tension and depression in according to narcissism and emotional immaturity.

Moreover it is possible to find in this subject an exaggerated need of kindnesses sometimes using manipulative strategies.

The Content Scales of Anxiety (ANX) and Health Worries (HEA) (Table 2) are also above the average and confirm the symptomatology revealed through the Basic Scales above mentioned.

\begin{tabular}{|l|l|l|}
\hline Content Scale & Mean Value & Standard Deviation \\
\hline ANX & 65.12 & 11.64 \\
\hline HEA & 65.25 & 13.17 \\
\hline
\end{tabular}

Table 2: Content scales with a mean value above the cut-off.

The Anxiety scale (ANX) is composed of 23 items and it is used to identify general problems connected to anxiety. All the subjects' present tachycardia, difficulties of falling asleep, worries, problems about keep their mind focused on something.

The Health Worries scale (HEA) is composed of 36 items concerning general deceases of physical nature. The symptomatology is spread equally to all the examined subjects and it is usually manifested in: gastrointestinal symptoms (vomit, nausea, constipation, gastralgia), neurological problems (dizziness, faintings), cardiovascular symptoms (tachycardia, palpitations), breathing difficulties (cough, dyspnea) and dermatological problems.

Analyzing the Supplementary Scales of Harris and Lingoes, the highest scores are those of the Harris and Lingoes Supplementary Scales D1, D3 and Hy3 (Table 3). The Supplementary Scale D1 is usually present in people who feel lack of energy facing particular situation of stress. The Supplementary Scale D3 underlines health and physical worries together with the Supplementary Scale Hy3 which underlines discomforts, weakness, and lack of concentration.

\begin{tabular}{|l|l|l|}
\hline $\begin{array}{l}\text { Harris and Lingoes } \\
\text { Supplementary Scales }\end{array}$ & Mean Value & Standard Deviation \\
\hline D1 & 67.41 & 12.73 \\
\hline D3 & 65.75 & 14.14 \\
\hline Hy3 & 66.66 & 11.04 \\
\hline
\end{tabular}

Table 3: Harris and Lingoes Supplementary Scales with a mean value above the cut-off (65).

These characteristics outline the neurotic style and are all very peculiar in this sample. The traits help to delineate the basic 
personalities with a particular focus on their common characteristics and their individual differences. With the word "trait" we mean a large way of feeling, interacting and thinking about oneself or the social background and the way an individual apply them in everyday social and personal situations. For this reason the traits are widely spread among the population and are not to be identified necessarily as pathological aspects. They complete the scheme that helps to represent one's personality, a group of recognizable characteristics that are usually shown in a broad range of situation during a long period of time.

In this study, the examined cases received the diagnosis of Adjustment Disorder, in some cases in addition to Anxiety and Depression (or anxiety alone) but no one can be classified as an individual with Personality Disorder (following the DSM-IV-TR classification).

Anyway, the common traits could be read as a group of actions and interactions that delineate the "neurotic style". In conclusion, the sectorial approach to the classification of the personality disorders underlines a discontinuity and a significant difference between what we intend to be "normal" or "disturbed". The dimensional approach assumes that a continuum between these two aspects exists: the individual differences are seen as incremental differences along this continuum.

It is more and more needed a new approach to psychiatric diagnosis in order to combine the clinical-methodological significance of the dimensional diagnosis with the simplicity and practicality of the diagnostic categories. In all the examined subjects, mobbing is presented as a source of incredible stress and it could have activated the somatization process that give the problem a substantial clinical value expressed in the forms of anxiety and depression. The presence of neurotic aspects in these personalities give a substrate of possible dysfunctional mechanisms in the adaptation process, especially when there's a clear presence of relevant and continuous stress factors, typical of mobbing, that slowly lead to a psychopathological suffering.

This is an observational study that provided preliminary data; therefore we did not apply a statistical elaboration to the results we've collected. Since our sample of patients is increasing over time and our observational study goes on, we will commit ourselves to a more detailed article in the near future which can include an appropriate statistical analysis, in order to further investigate this phenomenon and its psychological implications on our ever-changing society.

\section{References}

1. Leymann H (1984) The silencing of a skilled technician. Working Environment 4: 236-238.

2. Zapf D, Einarsen S (2001) Bullying in the workplace: recent trends in research and practice-an introduction. Eur $\mathrm{J}$ Work and Organizat Psychol 10: 369-73.

3. Gandolfo R (1995) MMPI-2 profiles of workers compensation claimants who present with complaints of harassment. Journal of Clinical Psychology 51: 711-715.

4. NolfeG, NolfeG, Petrella C, Blasi F, Zontini G (2007) Psychopathological Dimensions of harassment in the workplace (mobbing). International Journal of Mental Health 4: 67-85.

5. Balducci C, Alfano V, Fraccaioli F (2009)Relationship between mobbing at work and MMPI-2 personality profile, posttraumatic stress symptoms, and suicidal ideation and behavior. Violence and Victims 24:52-67.

6. Meehl PE, Hathaway SR (1946) The K factor as a suppressor variable in the Minnesota Multiphasic Personality Inventory. Journal of Applied Psychology 30: 525-564.

7. Butcher JN, Williams CL (2007)Fondamenti per l'interpretazione del MMP-2 e del MMPI-A, organizzazionispeciali, Firenze.

8. David L, Thomas W (1979) A Validating clinicians' hunches: Construction of a new MMPI critical item set. In Journal of Consulting and Clinical Psychology47: 277-284.

9. Koss MP, Butcher JN (1973)a comparison of psychiatric patients' selfreports with other sources of clinical information. Journal of Research in Personality 7: 225-236.

10. Wiener DN, Harmon LR (1946) Subtle and obvious keys for the MMPI. Their development. Advisement Bullettin No.16, Regional Veterans Administration Office, Minneapolis, MN.

11. Gough Harrison G (1950)The F minus K dissimulation index for the Minnesota Multiphasic Personality Inventory. Journal of Consulting Psychology 14: 408-413.

12. Richard R, Kenneth SW, Mary MA, Michael VJ (2003) Detection of Feigned Mental Disorders A Meta-Analysis of the MMPI-2 and Malingering. Assessment 1:21. 